\title{
Quadrupolar Dyes Based on Highly Polarized Coumarins
}

\author{
Krzysztof Górski, Irena Deperasińska, Glib V. Baryshnikov, Shuhei Ozaki, Kenji Kamada,* Hans Ågren,*
} and Daniel T. Gryko*

Cite This: Org. Lett. 2021, 23, 6770-6774

Read Online

\section{ACCESS \\ Wlll Metrics \& More \\ Article Recommendations \\ Supporting Information}

ABSTRACT: The fluorescence and other photophysical parameters of highly polarized, quadrupolar bis-coumarins possessing an electron-rich pyrrolo[3,2-b]pyrrole bridging unit are highly dependent on the linking position between both chromophores. Delocalization of the LUMO on the entire $\pi$-system results in intense emission and strong two-photon absorption.

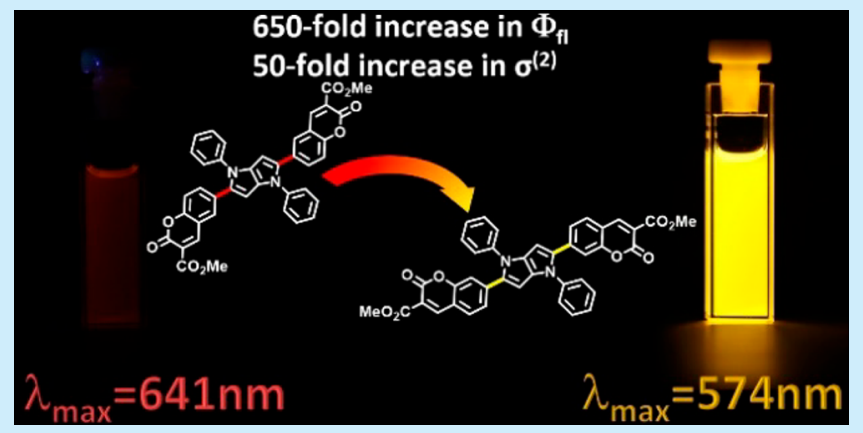

oumarins are a large family of oxygen-containing heterocycles which were first isolated 200 years ago. Since then, interest in this class of substances has remained strong owing to the many derivatives that display broad biological activity. ${ }^{1,2}$ Coumarins have also attracted significant scientific attention due to their unique photophysical properties. $^{3-5}$ Their simple synthesis combined with the relative ease of functionalization makes it possible to create a wide range of dyes. $^{6}$ A particularly important class of coumarin-based emitters are donor-acceptor systems possessing an electrondonating substituent at position 7 and an electron-withdrawing one at position $3 .^{7,8}$ It is well-known that 7 -aminocoumarins exhibit excellent emissive properties. ${ }^{9}$ Intriguingly, switching the position of an electron-donating substituent from 7 to 6 leads to marked differences in optoelectronic properties. ${ }^{10-14}$ In the case of 6-aminocoumarins the emission is weak and redshifted in comparison with the corresponding 7-aminocoumarins. $^{15,16}$ In this letter we address a key question: is this a general effect of electron-donating substituents irrespective of their structure? To answer this query we adopted a pyrrolo[3,2-b]pyrrole (PP) scaffold as the bridge between two coumarins. It was chosen because of its exceptional electron-donating character. At the same time, the newly designed dyes constitute the first quadrupolar, centrosymmetric, acceptor-donor-acceptor (A-D-A) architecture that possesses coumarin units, which can be an excellent two-photon absorber. ${ }^{13,17}$

Numerous studies have shown that in pyrrolo[3,2-b]pyrroles the electronic communication is particularly strong at positions 2 and $5 .^{18}$ In order to install coumarin units into these locations on this heterocycle, formyl-coumarin derivatives were designed for the recently optimized multicomponent reaction between aromatic amines, butane-2,3-dione, and aromatic aldehydes for the pyrrolo[3,2-b] pyrrole synthesis. ${ }^{19}$ The formyl-coumarin derivatives were additionally designed to possess an auxiliary $\mathrm{CO}_{2} \mathrm{Me}$ group at position 3 in order to increase their accepting character. The isomeric 6- and 7formyl coumarins $\mathbf{2 a}$ and $\mathbf{2 b}$ designed for this purpose are shown in Scheme 1.

The first attempts at performing the Knoevenagel condensation between dialdehyde $\mathbf{1 a}$ and dimethyl malonate in methanol did not give the desired product 2a. Due to the presence of two aldehyde groups in compound 1a, a significant portion of the isolated material was the double condensation product. Subsequent experiments finally made it possible to obtain 2a in satisfactory yield (68\%) through the use of isopropanol as the reaction medium in which the solubility of $\mathbf{2 a}$ is significantly lower than in methanol, leading to the precipitation of the desired product from the reaction mixture.

The last step in the synthetic pathway leading to regioisomeric $\mathrm{A}-\mathrm{D}-\mathrm{A}$ systems was the multicomponent condensation between $\mathbf{2 a}$ and $\mathbf{2 b}$, 4-decylaniline, and butane2,3-dione in the presence of a catalytic amount of iron(III) perchlorate. Thus, Coum6 with a yield of 9\%, and Coum7 with a yield of $17 \%$ were obtained (Scheme 1). The 2 -fold higher yield of Coum7 is probably the result of the orientation of the formyl group in the aldehyde $\mathbf{2} \mathbf{b}$ being in the para position to the malonylidene subunit, which increases the electron deficiency within the formyl group, enhancing its

Received: July 14, 2021

Published: August 16, 2021 
Scheme 1. Synthesis of Bis(coumarin)pyrrolopyrroles Coum6 and Coum7

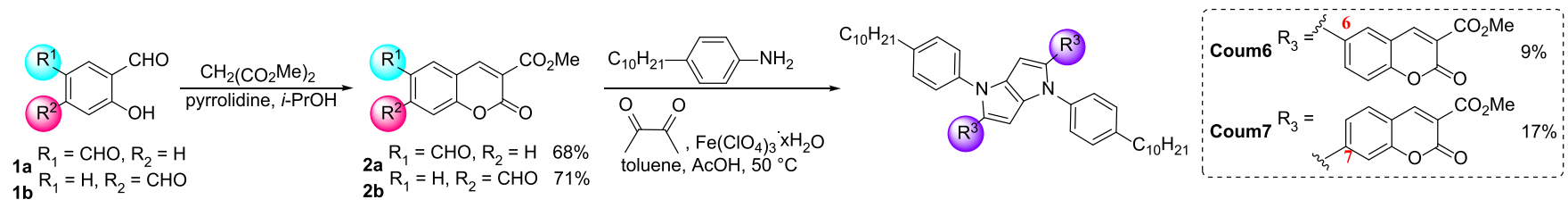

reactivity toward the nucleophiles. To interrogate the role of the bridging position on the photophysical properties, absorption and emission measurements were carried out in three solvents differing in polarity (Figures 1 and 2, Table 1).

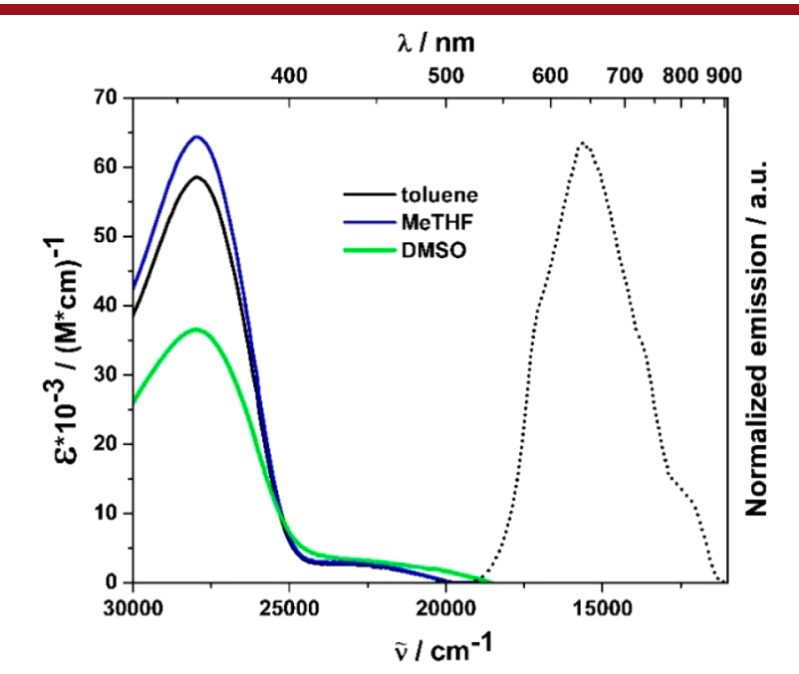

Figure 1. Absorption (solid line) and normalized fluorescence (dotted line) spectra of Coum6 measured in three different solvents recorded with excitation at $354 \mathrm{~nm}$. Legend specifies colors of lines.

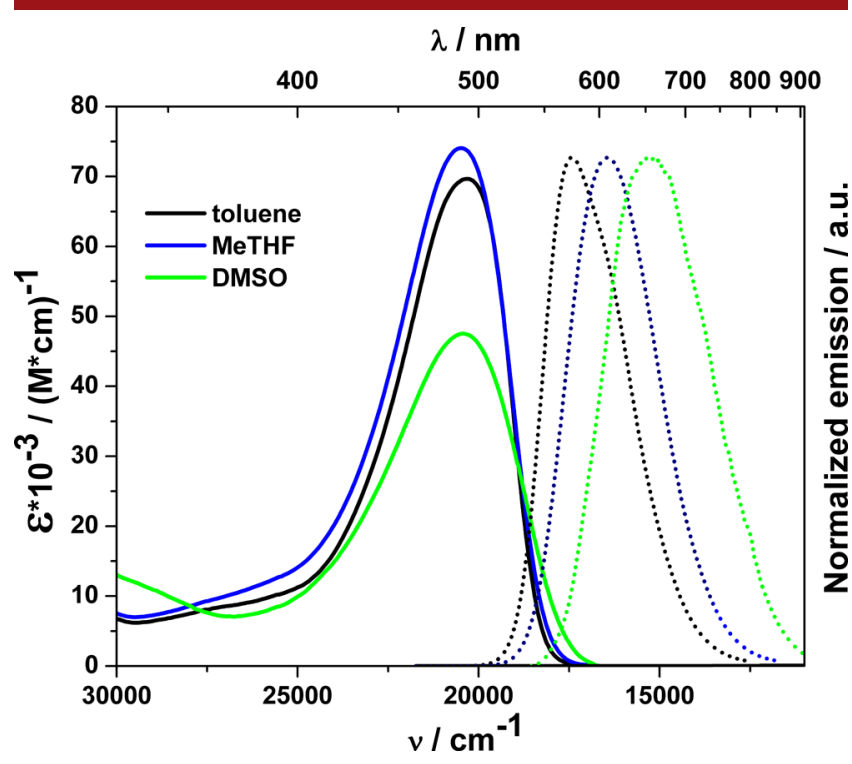

Figure 2. Absorption (solid line) and normalized fluorescence (dotted line) spectra of Coum7 measured in three different solvents recorded with excitation at $440 \mathrm{~nm}$. Legend specifies colors of lines.

The differences in the spectroscopic properties of isomeric A$\mathrm{D}-\mathrm{A}$ dyes become visible by the naked eye (Coum6 - orange solid, Coum7 - red solid). Coum6 exhibits intense UV light absorption $\left(\lambda_{\text {abs }}=358 \mathrm{~nm}\right)$, whereas in the low-energy part of the absorption spectrum a weak, broad band can be observed
Table 1. Photophysical Data of Coum6 and Coum7 Measured in Solution

\begin{tabular}{clcccc} 
Dye & Solvent & $\lambda_{\text {abs }}{ }^{\max }[\mathrm{nm}]$ & $\lambda_{\mathrm{em}}{ }^{\max }[\mathrm{nm}]$ & $\Phi_{\mathrm{fl}}[\%]$ & $\Delta \nu\left[\mathrm{cm}^{-1}\right]$ \\
Coum6 & toluene & 358,439 & 641 & $0.06^{a}$ & 7200 \\
& MeTHF & 358,439 & - & - & - \\
\multirow{4}{*}{ Coum7 } & DMSO & 358,440 & - & - & - \\
& toluene & 486 & 574 & $39^{b}$ & 3000 \\
& MeTHF & 485 & 608 & $30^{b}$ & 4100 \\
& DMSO & 487 & 649 & $0.02^{b}$ & 5300
\end{tabular}

${ }^{a}$ Reference: 9,10-diphenylantracene in toluene $\left(\Phi_{\mathrm{fl}}=0.70\right)$. ${ }^{b}$ Reference: Coumarin 153 in toluene $\left(\Phi_{\mathrm{fl}}=0.40\right)$.

$\left(\lambda_{\mathrm{abs}}=440 \mathrm{~nm}\right)$. The opposite effect occurs in the case of Coum7, where strong absorption of yellow light $\left(\lambda_{\mathrm{abs}}=485\right.$ $\mathrm{nm})$ is accompanied by a residual absorption of UV radiation. In accordance with the centrosymmetric architecture, there is no solvatochromism in these dyes; however, a significant drop of absorption coefficient is observed while the polarity of the solvent increases (Figures 1 and 2).

There are two strong analogies between the above-described characteristics and photophysics of simpler D-A coumarins. First, in Coum6 the Stokes shift is large whereas there is only a moderate difference between the absorption and emission in the case of Coum7. Moreover, for Coum6 a very weak red fluorescence is observed, while Coum7 has strong emission (Table 1). It should also be mentioned that, for more polar solvents, the Coum6 emission is below the detection limit. On the other hand, changing the substitution position on the coumarin subunit from 6 to 7 results in a 650 -fold increase in the fluorescence quantum yield from $0.06 \%$ to $39 \%$ in toluene. Due to the incomparably stronger emissive properties, in the case of Coum7, solvatofluorochromism can be observed indicating excited-state symmetry-breaking. ${ }^{20}$ The successive increase in the solvent polarity results in a clear Stokes shift, from $3000 \mathrm{~cm}^{-1}$ in toluene to $5300 \mathrm{~cm}^{-1}$ in DMSO. The described phenomenon is also accompanied by a significant decrease of the fluorescence quantum yield, down to $0.02 \%$ in DMSO.

In principle the photophysical properties of these quadrupolar bis-coumarins mirror the properties of 7-aminocoumarins vs 6-aminocoumarins; i.e., coumarins possessing electron-donating groups at the 6-position have weak but bathochromically shifted emission whereas coumarins substituted at the 7-position exhibit strong emission.

From a purely structural perspective the investigated dyes can be considered as bis-coumarins and, at the same time, as centrosymmetric pyrrolo[3,2-b]pyrroles. The photophysical properties of Coum 6 and Coum 7 can be directly compared to bis-2,5-(4-cyanophenyl)pyrrolo[3,2-b]pyrrole, ${ }^{18}$ which is the prototypical A-D-A pyrrolo[3,2-b]pyrrole. Absorption of Coum 7 is bathochromically shifted by ca. $80 \mathrm{~nm}$ and the emission by over $120 \mathrm{~nm}$, which reveals that the coumarin scaffolds affect the electron structure making it a truly $\pi$ expanded system. On the other hand, the main absorption 
band of Coum6 is hypsochromically shifted ca. $50 \mathrm{~nm}$ in comparison to bis-2,5-(4-cyanophenyl)pyrrolo[3,2-b]pyrrole.

The investigated dyes $\mathrm{A}-\mathrm{D}-\mathrm{A}$ architecture encouraged us to explore their two-photon absorption (TPA) properties, as $\mathrm{A}-\mathrm{D}-\mathrm{A}$ is one of the most successful motifs of TPA dyes. The TPA spectra were measured using a femtosecond openaperture Z-scan method ${ }^{21,22}$ in a MeTHF (Figure 3). Coum7

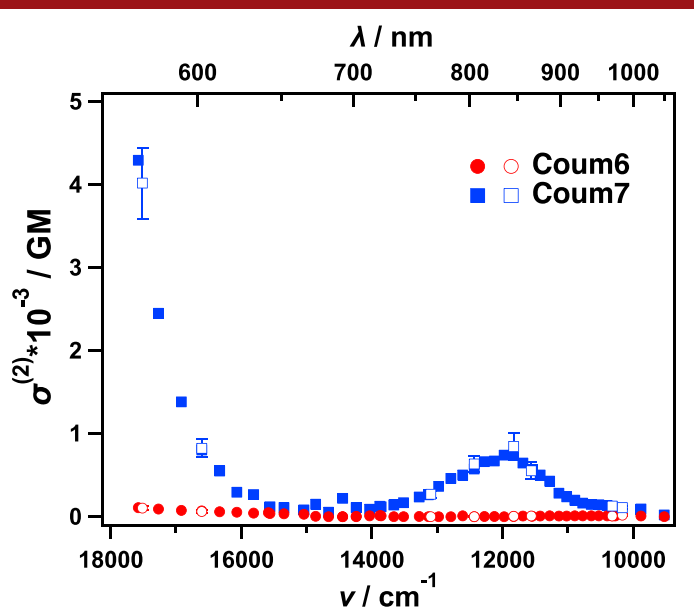

Figure 3. Two-photon absorption spectra of Coum6 (red circles) and Coum7 (blue squares) both in MeTHF. Solid symbols represent data measured at fixed excitation powers. Contoured symbols with error bars show data obtained through confirming its excitation intensity dependence.

was found to have a strong and broad TPA peak centered at $12000 \mathrm{~cm}^{-1}(840 \mathrm{~nm})$ with the peak TPA cross section equal to $850 \pm 160 \mathrm{GM}$, where $1 \mathrm{GM}=10^{-50} \mathrm{~cm}^{4} \mathrm{~s}$ photon ${ }^{-1}$ molecule ${ }^{-1}$. The two-photon absorption cross section increased even further starting from $650 \mathrm{~nm}$ toward the blue edge of the spectrum, while the $\sigma^{(2)}$ reached $4000 \pm 430 \mathrm{GM}$ at $17500 \mathrm{~cm}^{-1}(453 \mathrm{~nm})$. In contrast, Coum 6 showed a much weaker TPA, with $\sigma^{(2)} s \leq 10 \mathrm{GM}$ for $9500-15000 \mathrm{~cm}^{-1}$ $(1050-660 \mathrm{~nm})$. The spectral magnitude was monotonically increased at the photon energy higher than $15000 \mathrm{~cm}^{-1}$ (Figure S17); nevertheless, the maximum value observed was $\sigma^{(2)}=100 \pm 26 \mathrm{GM}$ at $17500 \mathrm{~cm}^{-1}(453 \mathrm{~nm})$, which is in 40 fold contrast to that of Coum7.

The TPA peak of Coum7 at $12000 \mathrm{~cm}^{-1}$ (i.e., $24000 \mathrm{~cm}^{-1}$ in the transition energy) did not match the transition energy produced by the one-photon absorption peak (Figure 2). This behavior can be understood using Laporte's selection rule complementary for one- and two-photon absorption. The significant increase observed at higher energies likely originates from a resonance enhancement. ${ }^{23}$

In order to investigate the effect of the coumarin substitution site on the photophysical properties, we combined our experimental results with TD-DFT calculations (including optimization of structures) using different functionals and basis sets (see Supporting Information (SI) for details). We note that the use of the standard TD DFT/B3LYP/6-31G(d,p) approach in the case of CT systems like Coum6 and Coum 7 is insufficient to properly reproduce the photophysical properties. To obtain better agreement between experiment and computational results, hybrid functionals with an increased amount of Hartree-Fock exchange, such as B3LYP-37, should be used. Calculated energies of electronic transitions with that functional are shown in Table 2. Small differences between
Table 2. PCM/B3LYP-37/6-31G(d,p) Calculation Results of Coum 6 and Coum7 Electronic Transitions in Toluene ${ }^{a}$

$\begin{array}{ccc}\text { Transition } & \text { Coum6 } \lambda[\mathrm{nm}](f) & \text { Coum7 } \lambda[\mathrm{nm}](f) \\ \mathrm{S}_{0} \rightarrow \mathrm{S}_{1} & 448(0.062) & 475(2.089) \\ \mathrm{S}_{0} \rightarrow \mathrm{S}_{2} & 448(0.000) & 397(0.000) \\ \mathrm{S}_{0} \rightarrow \mathrm{S}_{3} & 342(1.914) & 354(0.051) \\ \mathrm{S}_{0} \rightarrow \mathrm{S}_{4} & 338(0.000) & 319(0.000) \\ \mathrm{S}_{0} \rightarrow \mathrm{S}_{5} & 338(0.055) & 315(0.000) \\ \mathrm{S}_{1} \rightarrow \mathrm{S}_{0} & 598(0.017) & 535(2.441)\end{array}$

${ }^{a}$ Wavelengths $(\lambda)$ and oscillator strengths (f) of the $S_{0} \rightarrow S_{i}$ electronic transitions.

experiment and calculations result from the limitations of the TD-DFT method and from the fact that molecules used in calculations do not possess alkyl chains at phenyl and carboxyl subunits.

As can be seen in the table, the oscillator strength of the $S_{0}$ $\rightarrow S_{1}$ transition in Coum6 is much lower $(f=0.062)$ compared to the $S_{0} \rightarrow S_{1}$ transition in Coum7 $(f=2.089)$ which indicates the effectively forbidden character of the Coum6 first electron transition, leading to a lack of absorption in the yellow region. On the other hand, the Coum7 absorption spectra are bathochromically shifted compared to Coum6, which is in line with the computational results. Moreover, the observed strong absorption in the UV range for Coum 6 corresponds to the allowed $S_{0} \rightarrow S_{3}$ transition $(f=$ 1.914). Due to the fact that the $S_{0} \rightarrow S_{1}$ as well as the $S_{1} \rightarrow S_{0}$ transitions are mainly described by HOMO/LUMO configurations, the two transitions possess similar properties; thus the forbidden nature of the $S_{0} \rightarrow S_{1}$ transition also manifests in the emissive properties of Coum6. However, an analysis of fluorescence spectra of investigated $\mathrm{A}-\mathrm{D}-\mathrm{A}$ systems indicates that, in the case of Coum6, a much larger Stokes shift is observed, compared to Coum7.

HOMOs of both Coum 6 and Coum 7 are mainly located on the electron-rich pyrrolo[3,2-b]pyrrole core (Figure 4). On the

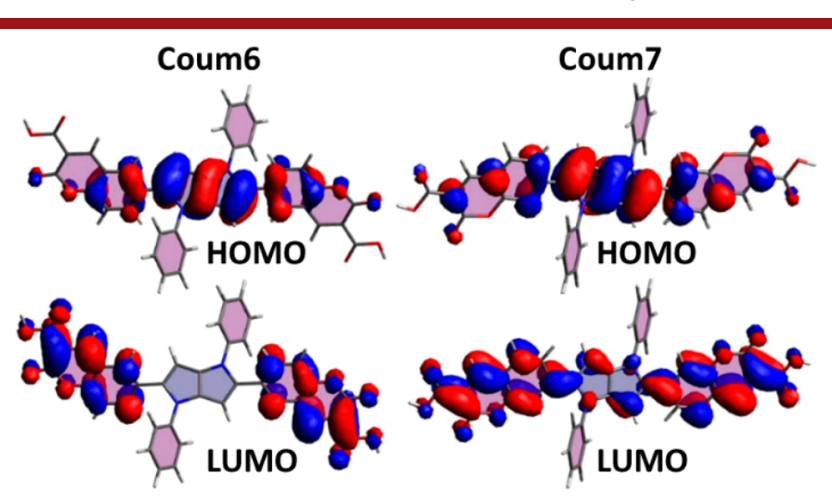

Figure 4. HOMO and LUMO orbitals of Coum6 and Coum7.

other hand, clear differences of the electron density can be observed for LUMOs. In the case of Coum6, the LUMO is completely located on the electron-accepting coumarin subunits, while the Coum7 LUMO orbital is also localized on the central pyrrolopyrrole core. Such shapes of the frontier orbitals indicate that the $S_{0} \rightarrow S_{1}$ transitions in Coum6 and Coum7 are intramolecular charge-transfer (CT) transitions from $\mathrm{D}$ to the two A centers, with a lesser degree of charge transfer in Coum7 (see SI for quantitative data). Moreover, upon excitation of Coum6, a better charge separation is observed (see SI for quantitative data). The larger charge 
separation in the Coum6 $S_{1}$ excited state leads to significant Coulomb interaction driven stabilization, manifested by the lower energy of the $S_{1} \rightarrow S_{0}$ transition and a significant drop in oscillator strength $(f=0.062)$ with regard to Coum7 $(f=$ 2.089). On the other hand, a much weaker charge separation in the Coum $7 S_{1}$ state, caused by the significant delocalization of the LUMO over both the pyrrolo[3,2-b] pyrrole core and the coumarin subunits, leads to a greater oscillator strength of the $\mathrm{S}_{1} \rightarrow \mathrm{S}_{0}$ transition.

A simulation of the TPA spectrum successfully reproduced the contrast features of Coum6 and Coum7 (Figure S5). For Coum 6 the transition intensity is weak for transitions up to $S_{8}$, with strong TPA transitions only existing for those to higher excited states. In contrast, for Coum7, a strong transitions to $\mathrm{S}_{2}$ appeared at $770 \mathrm{~nm}$, which corresponds to the experimentally observed TPA peak centered at $840 \mathrm{~nm}$ (Figure 3 ), though it is energetically overestimated. Calculation results also show complementary behavior of one- and two-photon transitions for these centrosymmetric molecules. Weak or no TPA transitions were observed to the excited states to which one-photon transitions are strong $\left(S_{0} \rightarrow S_{3}\right.$ for Coum6 and $S_{0}$ $\rightarrow S_{1}$ for Coum 7$)$ as well as vice versa $\left(S_{0} \rightarrow S_{2}\right.$ of Coum 7 is a strong TPA transition but forbidden for one-photon absorption).

By applying the concept of electron donor-acceptor systems, ${ }^{24}$ the differences between the transition energies and oscillator strengths for the excitation and fluorescence of Coum 6 and Coum 7 can be assigned to the differentiation of short-range interactions within the $\mathrm{AD}$ junction. This is in line with the results of Liu and co-workers for 6-aminocoumarins. ${ }^{25}$ The calculated solvent shifts of the transition energies, along with the increase of solvent polarity, are in good agreement with those observed (see SI). There is however no clear explanation for the observed decrease of fluorescence yield of Coum7 in polar DMSO. This suggests an opening of the nonradiative decay pathway and requires the use of increasingly sophisticated models of solvent effects for CT systems. $^{26-28}$

Both the quadrupolar architecture of the hybrid dyes and the bridging position of the coumarin scaffolds with the electronrich pyrrolo[3,2-b]pyrrole unit play decisive roles in the optoelectronic properties of the new dyes. In analogy to the classical 7-dialkylaminocoumarins, the emission of the quadrupolar bis-coumarin with a pyrrolo[3,2-b]pyrrole unit at the seventh position is strong and moderately bathochromically shifted. Shifting the bridge to the position 6 drastically changes the nature of the LUMO, resulting in its localization solely on the coumarin subunits. This results in a weakly emitting dye with $\lambda_{\mathrm{em}}{ }^{\max }$ at $650 \mathrm{~nm}$. Marked differences in their two-photon absorbing properties (TPA cross-section in the near-infrared region decreased from $850 \mathrm{GM}$ for Coum7 to less than $10 \mathrm{GM}$ for Coum6) are also caused by the diversity of the LUMO distribution.

\section{ASSOCIATED CONTENT}

\section{Supporting Information}

The Supporting Information is available free of charge at https://pubs.acs.org/doi/10.1021/acs.orglett.1c02349.

Quantum chemical calculation and photophysical experimental data, and synthetic procedures, as well as ${ }^{1} \mathrm{H}$ and ${ }^{13} \mathrm{C}\left\{{ }^{1} \mathrm{H}\right\}$ NMR spectra (PDF)

\section{AUTHOR INFORMATION}

\section{Corresponding Authors}

Kenji Kamada - Nanomaterials Research Institute (NMRI), National Institute of Advanced Industrial Science and Technology (AIST), Ikeda, Osaka 563-8577, Japan; Department of Chemistry, Graduate School of Science and Technology, Kwansei Gakuin University, Sanda 669-1337, Japan; Oorcid.org/0000-0002-7431-5254; Email: k.kamada@aist.go.jp

Hans Ågren - Department of Physics and Astronomy, Uppsala University, SE-751 20 Uppsala, Sweden; 이이.org/00000002-1763-9383; Email: hagren@kth.se

Daniel T. Gryko - Institute of Organic Chemistry, Polish Academy of Sciences, 01-224 Warsaw, Poland; (1) orcid.org/ 0000-0002-2146-1282; Email: dtgryko@icho.edu.pl

\section{Authors}

Krzysztof Górski - Institute of Organic Chemistry, Polish Academy of Sciences, 01-224 Warsaw, Poland; 10 orcid.org/ 0000-0002-6439-2651

Irena Deperasińska - Institute of Physics, Polish Academy of Sciences, 02-668 Warsaw, Poland

Glib V. Baryshnikov - Laboratory of Organic Electronics, Department of Science and Technology, Linköping University, SE-60174 Norrköping, Sweden; 다이.org/0000-00020716-3385

Shuhei Ozaki - Nanomaterials Research Institute (NMRI), National Institute of Advanced Industrial Science and Technology (AIST), Ikeda, Osaka 563-8577, Japan; Department of Chemistry, Graduate School of Science and Technology, Kwansei Gakuin University, Sanda 669-1337, Japan

Complete contact information is available at:

https://pubs.acs.org/10.1021/acs.orglett.1c02349

\section{Notes}

The authors declare no competing financial interest.

\section{ACKNOWLEDGMENTS}

This work was financially supported by the Foundation for Polish Science (TEAM POIR.04.04.00-00-3CF4/16-00). We also thank Global Research Laboratory Program (2014K1A1A2064569) through the National Research Foundation (NRF) funded by Ministry of Science, ICT \& Future Planning (Korea) and the National Science Centre, Poland, under QuantERA programme, Project 2017/25/Z/ST2/ 03038. G.V.B. acknowledges the financial support of the Swedish Research Council (Starting Grant No. 2020-04600). The quantum-chemical calculations were performed with computational resources provided by Swedish National Infrastructure for Computing (SNIC 2020-3-29) at the High-Performance Computing Center North (HPC2N) partially funded by the Swedish Research Council through the Grant Agreement No. 2018-05973.Theoretical calculations were also performed at the Interdisciplinary Centre of Mathematical and Computer Modelling (ICM) of the Warsaw University under the computational grant G-32-10. This work was partially supported by JSPS KAKENHI Grant Number $21 \mathrm{H} 01887(\mathrm{KK})$. 


\section{REFERENCES}

(1) Stefanachi, A.; Leonetti, F.; Pisani, L.; Catto, M.; Carotti, A. Coumarin: A Natural, Privileged and Versatile Scaffold for Bioactive Compounds. Molecules 2018, 23 (2), 250.

(2) Srikrishna, D.; Godugu, C.; Dubey, P. K. A Review on Pharmacological Properties of Coumarins. Mini-Rev. Med. Chem. 2018, 18 (2), 113-141.

(3) Tsukamoto, K.; Shinohara, Y.; Iwasaki, S.; Maeda, H. A Coumarin-Based Fluorescent Probe for $\mathrm{Hg} 2+$ and $\mathrm{Ag}+$ with an $\mathrm{N}^{\prime}$ Acetylthioureido Group as a Fluorescence Switch. Chem. Commun. 2011, 47 (17), 5073-5075.

(4) Adronov, A.; Gilat, S. L.; Fréchet, J. M. J.; Ohta, K.; Neuwahl, F. V. R.; Fleming, G. R. Light Harvesting and Energy Transfer in LaserDye-Labeled Poly(Aryl Ether) Dendrimers. J. Am. Chem. Soc. 2000, 122 (6), 1175-1185.

(5) Liu, X.; Xu, Z.; Cole, J. M. Molecular Design of UV-Vis Absorption and Emission Properties in Organic Fluorophores: Toward Larger Bathochromic Shifts, Enhanced Molar Extinction Coefficients, and Greater Stokes Shifts. J. Phys. Chem. C 2013, 117 (32), 16584-16595.

(6) Bassolino, G.; Nançoz, C.; Thiel, Z.; Bois, E.; Vauthey, E.; Rivera-Fuentes, P. Photolabile Coumarins with Improved Efficiency through Azetidinyl Substitution. Chem. Sci. 2018, 9 (2), 387-391.

(7) Jones, G.; Rahman, M. A. Fluorescence Properties of Coumarin Laser Dyes in Aqueous Polymer Media. Chromophore Isolation in Poly(Methacrylic Acid) Hypercoils. J. Phys. Chem. 1994, 98 (49), 13028-13037.

(8) Kielesiński, Ł.; Morawski, O.; Dobrzycki, Ł.; Sobolewski, A. L.; Gryko, D. T. The Coumarin-Dimer Spring-The Struggle between Charge Transfer and Steric Interactions. Chem. - Eur. J. 2017, 23 (38), 9174-9184.

(9) Grandberg, I. I.; Denisov, L. K.; Popova, O. A. 7-Aminocoumarins (Review). Chem. Heterocycl. Compd. 1987, 23 (2), 117142.

(10) Samanta, A.; Fessenden, R. W. Excited-State Dipole Moment of 7-Aminocoumarins as Determined from Time-Resolved Microwave Dielectric Absorption Measurements. J. Phys. Chem. A 2000, 104 (37), 8577-8582.

(11) Krystkowiak, E.; Dobek, K.; Maciejewski, A. An Intermolecular Hydrogen-Bonding Effect on Spectral and Photophysical Properties of 6-Aminocoumarin in Protic Solvents. Photochem. Photobiol. Sci. 2013, 12 (3), 446-455.

(12) Lin, Q.; Bao, C.; Fan, G.; Cheng, S.; Liu, H.; Liu, Z.; Zhu, L. 7Amino Coumarin Based Fluorescent Phototriggers Coupled with Nano/Bio-Conjugated Bonds: Synthesis, Labeling and Photorelease. J. Mater. Chem. 2012, 22 (14), 6680-6688.

(13) Klausen, M.; Dubois, V.; Clermont, G.; Tonnelé, C.; Castet, F.; Blanchard-Desce, M. Dual-Wavelength Efficient Two-Photon Photorelease of Glycine by $\pi$-Extended Dipolar Coumarins. Chem. Sci. 2019, 10 (15), 4209-4219.

(14) Shin, J.; Verwilst, P.; Choi, H.; Kang, S.; Han, J.; Kim, N. H.; Choi, J. G.; Oh, M. S.; Hwang, J. S.; Kim, D.; et al. Harnessing Intramolecular Rotation To Enhance Two-photon Imaging of $\mathrm{A} \beta$ Plaques through Minimizing Background Fluorescence. Angew. Chem., Int. Ed. 2019, 58 (17), 5648-5652.

(15) Krystkowiak, E.; Maciejewski, A. Changes in Energy of Three Types of Hydrogen Bonds upon Excitation of Aminocoumarins Determined from Absorption Solvatochromic Experiments. Phys. Chem. Chem. Phys. 2011, 13 (23), 11317-11324.

(16) Krystkowiak, E.; Dobek, K.; Burdziński, G.; Maciejewski, A. Radiationless Deactivation of 6-Aminocoumarin from the S1-ICT State in Nonspecifically Interacting Solvents. Photochem. Photobiol. Sci. 2012, 11 (8), 1322-1330.

(17) Kamada, K.; Iwase, Y.; Sakai, K.; Kondo, K.; Ohta, K. Cationic Two-Photon Absorption Chromophores with Double- and TripleBond Cores in Symmetric/Asymmetric Arrangements. J. Phys. Chem. C 2009, 113 (27), 11469-11474.

(18) Krzeszewski, M.; Thorsted, B.; Brewer, J.; Gryko, D. T. Tetraaryl-, Pentaaryl-, and Hexaaryl-1,4-Dihydropyrrolo[3,2- $b]$ -
Pyrroles: Synthesis and Optical Properties. J. Org. Chem. 2014, 79 (7), 3119-3128.

(19) Tasior, M.; Vakuliuk, O.; Koga, D.; Koszarna, B.; Górski, K.; Grzybowski, M.; Kielesiński, Ł.; Krzeszewski, M.; Gryko, D. T. Method for the Large-Scale Synthesis of Multifunctional 1,4-DihydroPyrrolo[3,2-b]Pyrroles. J. Org. Chem. 2020, 85 (21), 13529-13543.

(20) Poronik, Y. M.; Baryshnikov, G. V.; Deperasińska, I.; Espinoza, E. M.; Clark, J. A.; Ågren, H.; Gryko, D. T.; Vullev, V. I. Deciphering the Unusual Fluorescence in Weakly Coupled Bis-Nitro-Pyrrolo[3,2b]Pyrroles. Commun. Chem. 2020, 3 (1), 190.

(21) Kamada, K.; Matsunaga, K.; Yoshino, A.; Ohta, K. TwoPhoton-Absorption-Induced Accumulated Thermal Effect on Femtosecond Z-Scan Experiments Studied with Time-Resolved ThermalLens Spectrometry and Its Simulation. J. Opt. Soc. Am. B 2003, 20 (3), 529-537.

(22) Sheik-Bahae, M.; Said, A. A.; Wei, T.-H.; Hagan, D. J.; van Stryland, E. W. Sensitive Measurement of Optical Nonlinearities Using a Single Beam. IEEE J. Quantum Electron. 1990, 26 (4), 760769.

(23) Kamada, K.; Ohta, K.; Iwase, Y.; Kondo, K. Two-Photon Absorption Properties of Symmetric Substituted Diacetylene: Drastic Enhancement of the Cross Section near the One-Photon Absorption Peak. Chem. Phys. Lett. 2003, 372 (3-4), 386-393.

(24) Nagakura, S. Excited States. In Excited States; Lim, E. C., Ed.; Academic: New York, 1975; pp 324-340.

(25) Liu, X.; Cole, J. M.; Xu, Z. Substantial Intramolecular Charge Transfer Induces Long Emission Wavelengths and Mega Stokes Shifts in 6-Aminocoumarins. J. Phys. Chem. C 2017, 121 (24), 1327413279.

(26) Grabowski, Z. R.; Rotkiewicz, K.; Rettig, W. Structural Changes Accompanying Intramolecular Electron Transfer: Focus on Twisted Intramolecular Charge-Transfer States and Structures. Chem. Rev. 2003, 103 (10), 3899-4032.

(27) Krauter, C. M.; Möhring, J.; Buckup, T.; Pernpointner, M.; Motzkus, M. Ultrafast Branching in the Excited State of Coumarin and Umbelliferone. Phys. Chem. Chem. Phys. 2013, 15 (41), 17846.

(28) Morawski, O. W.; Kielesiński, Ł.; Gryko, D. T.; Sobolewski, A. L. Highly Polarized Coumarin Derivatives Revisited: SolventControlled Competition Between Proton-Coupled Electron Transfer and Twisted Intramolecular Charge Transfer. Chem. - Eur. J. 2020, 26 (32), 7281-7291. 